\title{
Forward energy flow and jet production in pp and pA collisions at the LHC with CMS
}

\author{
Merijn H F van de Klundert on behalf of the CMS Collaboration* \\ DESY (Germany) \\ E-mail: mvandeklecern.ch
}

\begin{abstract}
A measurement of inclusive differential cross sections for very forward jet production is presented as a function of jet energy in proton-lead collisions. These collisions were recorded with the CASTOR calorimeter of the CMS experiment at the LHC, which measures in the pseudorapidity range $-6.6<\eta<-5$.2. Proton beams with $4 \mathrm{TeV}$ and beams of lead nuclei with an energy of $1.58 \mathrm{TeV}$ per nucleon were used, corresponding to a center-of-mass energy per nucleon pair of $\sqrt{s_{\mathrm{NN}}}=5.02 \mathrm{TeV}$. Cross sections with either the proton $(\mathrm{p}+\mathrm{Pb})$ or the ion $(\mathrm{Pb}+\mathrm{p})$ travelling towards CASTOR are studied and unfolded to the stable particle level for jets with $p_{\mathrm{T}} \geq 3 \mathrm{GeV}$. The ratio $\mathrm{p}+\mathrm{Pb} / \mathrm{Pb}+\mathrm{p}$ is also presented. The measurements are compared to various event generators, and discussed in terms of the saturation of gluon densities at low fractional parton momenta. None of the event generators considered in this analysis are able to describe all the spectra in their full energy range, and deviations between data and the models in excess of two orders of magnitude are observed.
\end{abstract}

39th International Conference on High Energy Physics

July 4-11 2018

Coex, Seoul, South Korea

\footnotetext{
*Speaker.
} 


\section{Introduction}

The effects of nonlinear parton interactions in hadrons are expected to become significant at large parton densities. At small parton momenta fractions $x$, it is expected that nonlinear gluon interactions lead to saturation of the gluon density, and the density needs to be evolved with a nonlinear evolution equation. Furthermore, it is expected that gluon saturation is enhanced in heavy ions, since the gluon density in a heavy ion is larger than in a proton.

The $x$-value of the parton can, to leading order in the strong coupling $\alpha_{s}$, be reconstructed from the jet and beam kinematics via $x=p_{\mathrm{T}} \cdot e^{-|\eta|} / \sqrt{s}$. In this equation $p_{\mathrm{T}}$ is the transversal momentum of the jet, $\eta$ represents its pseudorapidity, and $\sqrt{s}$ is the collision center-of-mass energy. Therefore, low $p_{\mathrm{T}}$ jets in the forward direction in proton-lead collisions provide an observable with optimal sensitivity to gluon saturation.

An analysis of differential jet cross sections as a function of the jet energy is presented in proton-lead collisions with a centre-of-mass energy of $\sqrt{s_{\mathrm{NN}}}=5.02 \mathrm{TeV}$. The jets have an equivalent $p_{\mathrm{T}} \geq 3 \mathrm{GeV}$ and are within $-6.6<\eta<-5.2$. Collisions with the proton propagating towards the negative $\eta$-hemisphere are referred to as $\mathrm{p}+\mathrm{Pb}$, while the reverse beam configuration is denoted $\mathrm{Pb}+\mathrm{p}$. It should be noted that the center-of-mass frame is boosted with respect to the laboratory frame, and all results presented here are in the laboratory frame.

\section{The CASTOR calorimeter at the CMS experiment}

The central feature of the CMS apparatus [1] is a superconducting solenoid of $6 \mathrm{~m}$ internal diameter, providing a magnetic field of $3.8 \mathrm{~T}$. Within the solenoid volume are a silicon pixel and strip tracker, a lead tungstate crystal electromagnetic calorimeter (ECAL), and a brass and scintillator hadron calorimeter (HCAL), each composed of a barrel and two endcap sections. Forward calorimeters (HF) extend the pseudorapidity coverage provided by the barrel and endcap detectors. HF provides coverage in the range $3.0<|\eta|<5.2$.

The CASTOR calorimeter is the key detector of this analysis. It is a non-compensating electromagnetichadronic sampling Cherenkov calorimeter, which measures in $-6.6<\eta<-5.2$. It should be noted that CASTOR has no $\eta$-segmentation, and consequently energy instead of $p_{\mathrm{T}}$ spectra are presented. Various aspects of the performance of CASTOR propagate into the uncertainty in the jet measurement [2]: the overall energy scale uncertainty $(15 \%)$, the alignment uncertainty $(\mathscr{O}(3 \mathrm{~mm}))$, and energy-dependent correction factors for the hadronic jets.

\section{Event selection and event generators}

Hadronic, non-diffractive events were selected by requiring activity in both HF calorimeters. Effects of pileup where mitigated by selecting bunch crossings with maximally one good primary vertex. A consistent event selection was adopted on generator level. The response of the CMS detector was simulated including the uncertainty in the position of CASTOR. To accommodate for the alignment uncertainty of CASTOR, simulations were performed with CASTOR shifted maximally inwards and outwards with respect to the beampipe.

The measurements are compared to various even generators. The EPOS-LHC [3] and 
QGSJETII-04 [4] generators are both based on Regge-Gribov theory and incorporate saturation. The HIJING [5] event generator is based on collinear factorisation and incorporates nuclear shadowing. In addition, two models based on hybrid factorisation are used to interpret the data. For these models the hard parton is modelled using collinear factorisation and DGLAP evolution, while the soft partons are modelled using an unintegrated pdf. The KATIE-KS model $[6,7]$ provides a framework in which the strength of the nonlinear interactions can be varied; this allows to assess the sensitivity of the observables w.r.t. gluon saturation. The AAMQS model [8] also incorporates nonlinear evolution and is based on the Colour Glass Condensate model.

\section{Results and conclusions}

The CASTOR jet spectra are unfolded using the d'Agostini iterative procedure as implemented in ROOUNFOLD [9], as outlined in [2]. In order to estimate the model uncertainty in the procedure, the unfolding was performed using EPOS-LHC, HIJING, and QGSJETII-04.

The final unfolded spectra with their uncertainties are presented in Fig. 1 for $\mathrm{p}+\mathrm{Pb}$ (left), $\mathrm{Pb}+\mathrm{p}$ (middle), and $\mathrm{p}+\mathrm{Pb} / \mathrm{Pb}+\mathrm{p}$ (right) together with model predictions. The predictions from KATIE-KS and $\mathrm{AAMQS}$ for $\mathrm{p}+\mathrm{Pb}$ are included separately in fig. 2 .
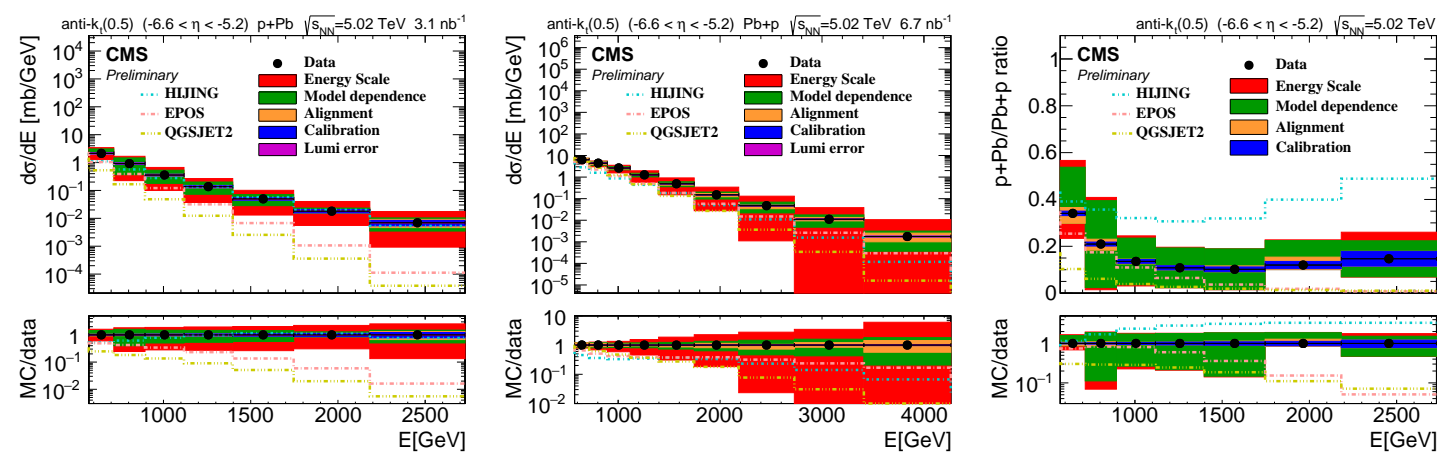

Figure 1: Differential jet energy cross sections for particle level jets in the CASTOR acceptance $(-6.6<$ $\eta<-5.2$ ). Model predictions are included for EPOS-LHC, HIJING, and QGSJETII-04. Left: p+Pb collisions. Middle: $\mathrm{Pb}+\mathrm{p}$ collisions . Right: the ratio of the cross sections for $\mathrm{p}+\mathrm{Pb} / \mathrm{Pb}+\mathrm{p}$. The figures are from [2].

The predictions of the EPOS-LHC and QGSJETII-04 models for $\mathrm{p}+\mathrm{Pb}$ collisions are clearly too soft, and underestimate the data by over two orders of magnitude at $2.5 \mathrm{TeV}$. The HIJING generator describes the data well.

The KATIE-KS predictions, presented in fig. 2, differ by an order of magnitude in the low energy region, while converging for the high energies, indicating that the CASTOR jet spectrum is indeed highly sensitive to saturation effects. The shape of the nonlinear predictions appears to describe the data best, while the linear predictions may have a better normalisation. The AAMQS model underestimates the data also in the region most affected by saturation.

For $\mathrm{Pb}+\mathrm{p}$ collisions all models underestimate the data for a few lower energy bins. From approx. 1.2 TeV onwards, all models are in agreement with the data within the systematic uncertainty.

The $\mathrm{p}+\mathrm{Pb} / \mathrm{Pb}+\mathrm{p}$ ratio, which has an improved resolution due to the partial cancelation of the scale uncertainty, is not described by any of the models. As a virtue of the error cancellation, the offset 
of HIJING, originating from the offset in the $\mathrm{Pb}+\mathrm{p}$ spectrum, is significant in the ratio.

The results establish that CASTOR jets constitute an observable with enhanced sensitivity to saturation effects, and none of the models under consideration describe all aspects of the data.

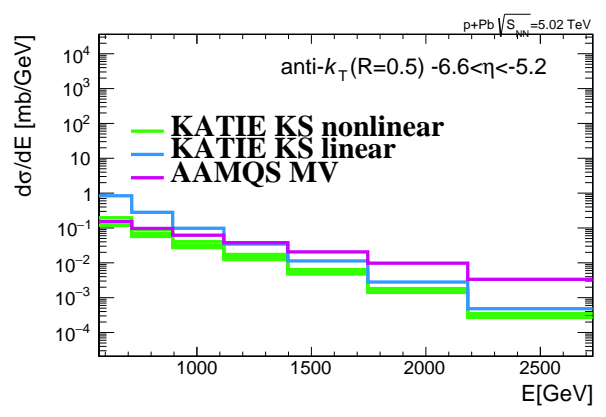

Figure 2: Differential jet energy cross sections for $\mathrm{p}+\mathrm{Pb}$ collisions in the CASTOR acceptance for the KATIE and AAMQS model (the figure has been included with permission from the authors).

\section{References}

[1] CMS Collaboration. The CMS experiment at the CERN LHC. JINST, 3:S08004, 2008. doi: 10.1088/1748-0221/3/08/S08004.

[2] CMS Collaboration. Very forward inclusive jet cross sections in $\mathrm{p}+\mathrm{Pb}$ collisions at $\sqrt{s_{\mathrm{NN}}}=$ 5.02 TeV. CMS-PAS-FSQ-17-001, CERN, 2017. URL https://cds.cern.ch/ record/2258273.

[3] T. Pierog, Iu. Karpenko, J. M. Katzy, E. Yatsenko, and K. Werner. EPOS LHC: Test of collective hadronization with data measured at the CERN Large Hadron Collider. Phys. Rev. C, 92: 034906, 2015. doi: 10.1103/PhysRevC.92.034906.

[4] S. Ostapchenko. QGSJET-II: Physics, recent improvements, and results for air showers. EPJ Web Conf., 52:02001, 2013. doi: 10.1051/epjconf/20125202001.

[5] X. Wang and M. Gyulassy. HIJING: A monte carlo model for multiple jet production in pp, pA, and AA collisions. Phys. Rev. D, 44:3501, 1991. doi: 10.1103/PhysRevD.44.3501.

[6] A. van Hameren. KaTie : For parton-level event generation with $k_{\mathrm{T}}$-dependent initial states. Comput. Phys. Commun., 224:371, 2018. doi: 10.1016/j.cpc.2017.11.005.

[7] K. Kutak and S. Sapeta. Gluon saturation in dijet production in $\mathrm{p}-\mathrm{Pb}$ collisions at Large Hadron Collider. Phys. Rev. D, 86:094043, 2012. doi: 10.1103/PhysRevD.86.094043.

[8] J. L. Albacete, P. Guerrero Rodríguez, and Y. Nara. Ultraforward particle production from color glass condensate and Lund fragmentation. Phys. Rev. D, 94:054004, 2016. doi: 10.1103/ PhysRevD.94.054004.

[9] T. Adye. Unfolding algorithms and tests using RooUnfold. May 2011. 